\title{
Evidence of prompt penetration electric fields during HILDCAA events
}

\author{
Regia Pereira Silva, Jose Humberto Andrade Sobral, Daiki Koga, and Jonas Rodrigues Souza \\ Instituto Nacional de Pesquisas Espaciais (INPE), São José dos Campos, 12227-010, São Paulo, Brazil \\ Correspondence to: Regia Pereira Silva (regiapereira@gmail.com, regia.pereira@inpe.br)
}

Received: 29 May 2017 - Revised: 15 September 2017 - Accepted: 18 September 2017 - Published: 27 October 2017

\begin{abstract}
High-intensity, long-duration continuous auroral electrojet (AE) activity (HILDCAA) events may occur during a long-lasting recovery phase of a geomagnetic storm. They are a special kind of geomagnetic activity, different from magnetic storms or substorms. Ionized particles are pumped into the auroral region by the action of Alfvén waves, increasing the auroral current system. The Dst index, however, does not present a significant downward swing as it occurs during geomagnetic storms. During the HILDCAA occurrence, the $\mathrm{AE}$ index presents an intense and continuous activity. In this paper, the response of Brazilian equatorial ionosphere is studied during three HILDCAA events that occurred in the year of 2006 (the descending phase of solar cycle 23) using the digisonde data located at São Luís, Brazil $\left(2.33^{\circ} \mathrm{S}, 44.2^{\circ} \mathrm{W}\right.$; dip latitude $\left.1.75^{\circ} \mathrm{S}\right)$. Geomagnetic indices and interplanetary parameters were used to calculate a cross-correlation coefficient between the $E_{y}$ component of the interplanetary electric field and the F2 electron density peak height variations during two situations: the first of them for two sets daytime and nighttime ranges, and the second one for the time around the pre-reversal enhancement (PRE) peak. The results showed that the pumping action of particle precipitation into the auroral zone has moderately modified the equatorial F2 peak height. However, F2 peak height seems to be more sensitive to HILDCAA effects during PRE time, showing the highest variations and sinusoidal oscillations in the cross-correlation indices.
\end{abstract}

Keywords. Ionosphere (equatorial ionosphere; ionospheremagnetosphere interactions; ionospheric disturbances)

\section{Introduction}

The conditions of the interplanetary environment are dominated by solar activity. During the maximum solar activity period some structures are more predominant, such as flares and coronal mass ejections (CMEs) (Wagner, 1984; Kahler, 1987; Webb and Howard, 2012). CMEs consist of huge numbers of energetic particles and magnetic energy release processes in the Sun, resulting in considerable effects in the Earth's ionosphere-thermosphere domain, eventually affecting satellite-borne instrumentation and ground electric power transmission networks. Once the CMEs with southward component of the interplanetary magnetic field (IMF) $B_{z}$ reach the Earth, magnetic storms occur suddenly, generating large disturbances in the magnetosphere-ionospherethermosphere system. It is well known when the IMF $B_{z}$ has a sudden southward turning can cause an eastward prompt penetration electric field during daytime and westward at nighttime (Kelley, 1989). Such an electric field is associated with an undershielding condition (Santos et al., 2016). A sudden northward turning causes the opposite effect which is associated with an overshielding condition. The equatorial Flayer dynamics moving up and down are controlled by the eastward and westward electric field, respectively.

During the declining phase of the solar cycle and the solar minimum period, another structure plays a major role. This structure is known as the corotating interaction regions (CIRs) (Smith and Wolfe, 1976; Watari, 1997; Gosling and Pizzo, 1999; Richardson, 2004). CIRs are created by the interaction of high-speed streams with upstream slow-speed streams. Despite the fact that CIRs may be not completely developed at $1 \mathrm{AU}$, one of their main features is the intense magnetic field, reaching $\sim 30 \mathrm{nT}$, while the regular values are 10-15 nT (Tsurutani et al., 1995; Gonzalez et al., 1999; 
Alves et al., 2006; Borovisky and Denton, 2006; Tsurutani et al., 2011a, b).

It has been known that the high-speed streams from corotating interaction regions are related to the occurrence of high-intensity, long-duration continuous auroral electrojet (AE) activities (HILDCAAs) (Tsurutani and Gonzalez, 1987; Sandanger et al., 2005; Tsurutani et al., 2006a, b; Kim, 2007; Hajra et al., 2014a, b, c). Therefore, HILDCAA events become more frequent when CIRs arise. HILDCAA takes place during a long recovery phase of Dst index, while interplanetary magnetic field (IMF) $B_{z}$ fluctuation amplitudes can reach around $\pm 10 \mathrm{nT}$. Some criteria are used to identify the phenomenon: (i) the $\mathrm{AE}$ index must reach an intensity peak greater than or equal to $1000 \mathrm{nT}$; (ii) the $\mathrm{AE}$ index needs to be almost continuous and never drop below $200 \mathrm{nT}$ for more than $2 \mathrm{~h}$ at a time; (iii) the event must have a duration of at least 2 days; and finally, and very important to mention, (iv) the phenomenon should take place outside the main phase of magnetic storms, i.e., during the recovery phase. It is worth mentioning that all criteria adopted to classify HILDCAA events were defined empirically. However, it is possible to consider a HILDCAA occurrence without strictly following all the criteria cited above (Tsurutani and Gonzalez, 1987, 1997; Tsurutani et al., 2004, 2006a; Sobral et al., 2006; Hajra et al., 2013).

Other key feature of HILDCAA events is a positive correlation between the AE index intensity and the Alfvénic fluctuations present in the $B_{z}$ component of the interplanetary magnetic field, which can be described by the expression $\delta V_{\mathrm{A}}=\delta B_{z} /\left(\mu_{o} n_{i} M_{i}\right)^{1 / 2}$, where $\delta B_{z}$ is the IMF $B_{z}$ fluctuation amplitude, $\mu_{o}$ the magnetic permeability, and $n_{i}$ and $M_{i}$ the ion density and mass, respectively. Since these fluctuations appear more frequently in fast-speed streams from coronal holes, its occurrence is more common in the descending phase and, secondly, in the solar minimum (Gonzalez et al., 2006; Kozyra et al., 2006; Guarnieri, 2006; Turner et al., 2006). Fluctuations of IMF through magnetic reconnection cause the transfer of mass, momentum, and energy of solar wind into the magnetosphere. The physical cause for the prolonged reduction of the Dst index is a continuous injection of plasmas in the ring current, which prevents the natural decay of the ring current. These injections occur even when the IMF $B_{z}$ is not constantly southward (Soraas et al., 2004; Kim, 2007). In addition to that mentioned above, HILDCAA is associated with the enhancement of magnetospheric relativistic electrons. Hajra et al. (2015) compared it to the isolated HILDCAA events. They assume that about $10-100 \mathrm{keV}$ electrons are injected into the inner magnetosphere during the events, so the anisotropic electrons generate electromagnetic chorus plasma waves constantly, and the chorus waves continuously accelerate the electron to $\mathrm{MeV}$ energies (Paulikas and Blake, 1979; Baker et al., 1986; Summers et al., 1998; Meredith et al., 2003; Tsurutani et al., 2006b, 2010).

Regarding the HILDCAA magnitude, the events may appear from weak to moderate. However, they can present very high emissions of photons per event, becoming more intense than some geomagnetic storms (Guarnieri, 2006). The important point here is concerned with the duration of the event, because even with a weak or moderate intensity, the photon emissions during HILDCAAs are observed almost constantly for several days, sometimes even weeks. For this reason, concerning the consequences in the equatorial and low-latitude ionosphere, the effects have reduced intensity.

The response of HILDCAA events to the equatorial ionosphere has been investigated in the South American sector. Sobral et al. (2006) studied the behavior of some ionospheric parameters over three equatorial-low-latitude stations on Brazil during three HILDCAA events in 2000 and 2001. Their results did not indicate evidence of prompt penetration electric fields; however, they noted that the ionospheric responses to disturbance dynamo and disturbed thermospheric winds during the events were similar to those observed during a typical storm event. Wei et al. (2008) announced that multiple electric field penetration to equatorial ionosphere is associated with HILDCAAs. This means that short pulses of dawn-dusk electric field bear the shielding effect. Koga et al. (2011) studied one 5-day-long HILDCAA event extracting prompt penetration drift effect. They compared F2 layer vertical drift with an empirical FS97 model (Fejer and Scherliess, 1997). They found a good agreement between F2 peak height and disturbance dynamo drift calculated by the model. The main objective of this paper is to investigate how the equatorial ionosphere in the Brazilian region behaves during three HILDCAA events, using a cross-correlation analysis between the $E_{y}$ component of the interplanetary electric field and the F2 peak height variation.

\section{Observational data and methodology}

In this paper we focus on the equatorial ionospheric response for three HILDCAA events occurring on the following days: 18-22 March (H-03), 6-11 June (H-06), and 18-26 December (H-12) in 2006 during the descending phase of solar cycle 23 . The numbers in parentheses refer to the month of the observation.

\subsection{Geomagnetic indices and interplanetary data}

The AE index, the SYM-H index, the solar wind speed (Vsw), and the $z$ component of the IMF $\left(B_{z}\right)$ from the OMNIWeb were used to classify the HILDCAA events, with $1 \mathrm{~min}$ resolution (http://omniweb.gsfc.nasa.gov/form/ omni_min.html). The solar radio flux data at $10.7 \mathrm{~cm}$, $2800 \mathrm{MHz}$, were obtained from the NOAA website (http: //spidr.ionosonde.net/spidr/) with 1-day resolution, and the $\mathrm{Kp}$ index data were obtained from the World Data Center for Geomagnetism, Kyoto, Japan (http://wdc.kugi.kyoto-u.ac.jp/ index.html). In this work the daily Kp sum value was used. 


\subsection{Digisonde data}

The ionospheric parameter used in this study was the electron density peak height of the $\mathrm{F}$ layer $(h m \mathrm{~F} 2)$. The data were obtained from the digisonde installed in São Luís, Maranhão, Brazil (geographic coordinates: $44.6^{\circ} \mathrm{W}, 2.33^{\circ} \mathrm{S}$; dip latitude $1.75^{\circ} \mathrm{S}$ ), with the temporal resolution of $15 \mathrm{~min}$. The $h m \mathrm{~F} 2$ data during HILDCAA events were analyzed and then compared with a set of 3-day averages belonging to a calm period ( $h m \mathrm{~F} 2$ 2quiet). This calm period was selected during a 15-day interval centered on a HILDCAA event, in which the daily sum Kp, $\Sigma \mathrm{Kp}$, was less than or equal to 24 . Thereafter, it was used a following ionospheric parameter for electron density peak height variation: $\Delta h m \mathrm{~F} 2=h m \mathrm{~F} 2-h m \mathrm{~F} 2$ _quiet. Every analysis in this work takes into account the $\Delta h m \mathrm{~F} 2$.

\subsection{Methodology}

The method applied in this study consists of a statistical analysis of the prompt penetration electric field effects on the ionosphere during HILDCAA events (Koga et al., 2011). The geoeffectiveness of the penetration of the interplanetary electric field (IEF) was identified by the correlation analysis between the $\mathrm{F} 2$ peak height variation $(\Delta h m \mathrm{~F} 2)$ and the $y$ component of the IEF $\left(E_{y}\right)$. Both $\Delta h m \mathrm{~F} 2$ and $E_{y}\left(E_{y}=\right.$ $-V_{x} \times B_{z}$ ) data were filtered with high-pass filter to allow only direct effects of electric field penetration, with a cutoff frequency of $9.26 \times 10^{-5} \mathrm{~Hz}(T=3 \mathrm{~h})$. This cutoff frequency was chosen to focus on short-lived electric fields, which lasts about $2 \mathrm{~h}$ and often is associated with southward and northward $B_{z}$ turnings. However, this cutoff frequency does not exclude the disturbance dynamo effects since, due to the inertia of the neutral air, a few hours are required to establish the disturbed wind system, and once established, the effects can last for several hours (Blanc and Richmond, 1980; Sastri et al., 1988; Abdu et al., 1995, 1997, 2006; Sobral et al., 1997; Richmond et al., 2003). Two time intervals were chosen to be representative of day and night periods; that is, 10 to $12 \mathrm{LT}$ represents daytime and 2 to $4 \mathrm{LT}$ represents nighttime. These time intervals have been established because they presented vertical drift peaks associated with the prompt penetration effects according to the empirical model of Fejer and Scherliess (1997). The Pearson correlation coefficients were calculated for these two periods of time maintaining fixed $\Delta h m \mathrm{~F} 2$ values and preceding the $E_{y}$ values for every $15 \mathrm{~min}$; that is, correlation values are calculated for delays of $\tau=0,15,30,45$, and $60 \mathrm{~min}$. The intent of doing this way using temporal delays rather than just a fixed value is to analyze whether there is a higher efficiency time between the $E_{y}$ penetration and the $h m \mathrm{~F} 2$ response.

The purpose of this method is to verify the correlation between the interplanetary/magnetospheric parameters and the ionospheric response in the equatorial region for each of the two periods of the day, during the HILDCAA occurrence.
Besides that, another analysis was done taking into account the time around the pre-reversal enhancement peak (PRE). The Pearson correlation coefficients were calculated for each hour from 17 to $23 \mathrm{LT}$. The purpose is to examine how the electric fields influence the PRE during the HILDCAA event.

\section{HILDCAA events}

Figures 1, 2, and 3 show the HILDCAA events analyzed in this work. The duration of HILDCAA events is indicated by a horizontal pink bar in the second panel from the top.

It is important to mention that the HILDCAA events chosen to this study were not considered for seasonal analysis. According to Hajra et al. (2013), in a study involving more than 100 events, no seasonal dependence was found.

The peak height of the F2 layer (red line), the quietday period reference (blue line), and $\Delta h m \mathrm{~F} 2$ (black line) are shown in the top panel of each figure. The $h m \mathrm{~F} 2$ _quiet reference value was estimated using $h m \mathrm{~F} 2$ data for three quiet days: 5 March $(\Sigma \mathrm{Kp}=2), 1$ April $(\Sigma \mathrm{Kp}=1+)$, and 2 April $(\Sigma \mathrm{Kp}=2)$ for $\mathrm{H}-03 ; 27$ May $(\Sigma \mathrm{Kp}=3+)$, 4 June $(\Sigma \mathrm{Kp}=3)$, and 23 June $(\Sigma \mathrm{Kp}=3+)$ for $\mathrm{H}-06$; and $2 \mathrm{De}-$ cember $(\Sigma \mathrm{Kp}=4), 4$ December $(\Sigma \mathrm{Kp}=1-)$, and 7 January $(\Sigma \mathrm{Kp}=2-)$ for $\mathrm{H}-12$.

Figure 1 regards $\mathrm{H}-03$. It may be seen that the $\mathrm{AE}$ index reached peak amplitude values above $1000 \mathrm{nT}$ five times during the event: on 19 March at 08:50 LT (1017 nT) and 18:55 LT (1006 nT) and on 21 March at 13:20 LT (1149 nT), 14:15 LT (1167 nT), and 14:45 LT (1092 nT). The $B_{z}$ fluctuations were around zero with amplitudes within $\pm 5 \mathrm{nT}$. The solar wind speed (Vsw) increased and remained at $700 \mathrm{~km} \mathrm{~s}^{-1}$ for several hours. The $E_{y}$ fluctuations were around +4 to $-4 \mathrm{mV} \mathrm{m}^{-1}$. The minimum value of SYM-H reached in the magnetic storm prior to the event was slightly less than $60 \mathrm{nT}$. Soon after, the SYM-H index shows that the HILDCAA event took place during the recovery phase of the magnetic storm, as marked by the horizontal bar in the second panel. The $h m \mathrm{~F} 2$ during the event is slightly higher than the average of the quiet days, except for the night of 20 March, in which from 18:00 LT the $h m F 2$ decreased $(\Delta h m \mathrm{~F} 2<0)$, i.e., the inhibition of PRE. The PRE is commonly responsible for brief and intense increase in zonal electric field, which leads to a height increase in the equatorial ionosphere and an increase in the growth rate of the generalized Rayleigh-Taylor instability (Abdu et al., 1981, 2003; Basu, 1997; Kelley et al., 2009). This instability is responsible for the equatorial spread-F and plasma bubble irregularity; however, these are not the focus of this work.

For the second event (H-06), although the auroral activities remained in high activity throughout the first 2 days, as shown in the third panel of Fig. 2, its peak value reached $1000 \mathrm{nT}$ only once, which was on 6 June at 16:15 LT $(1035 \mathrm{nT})$. The $B_{z}$ fluctuated significantly during most of 


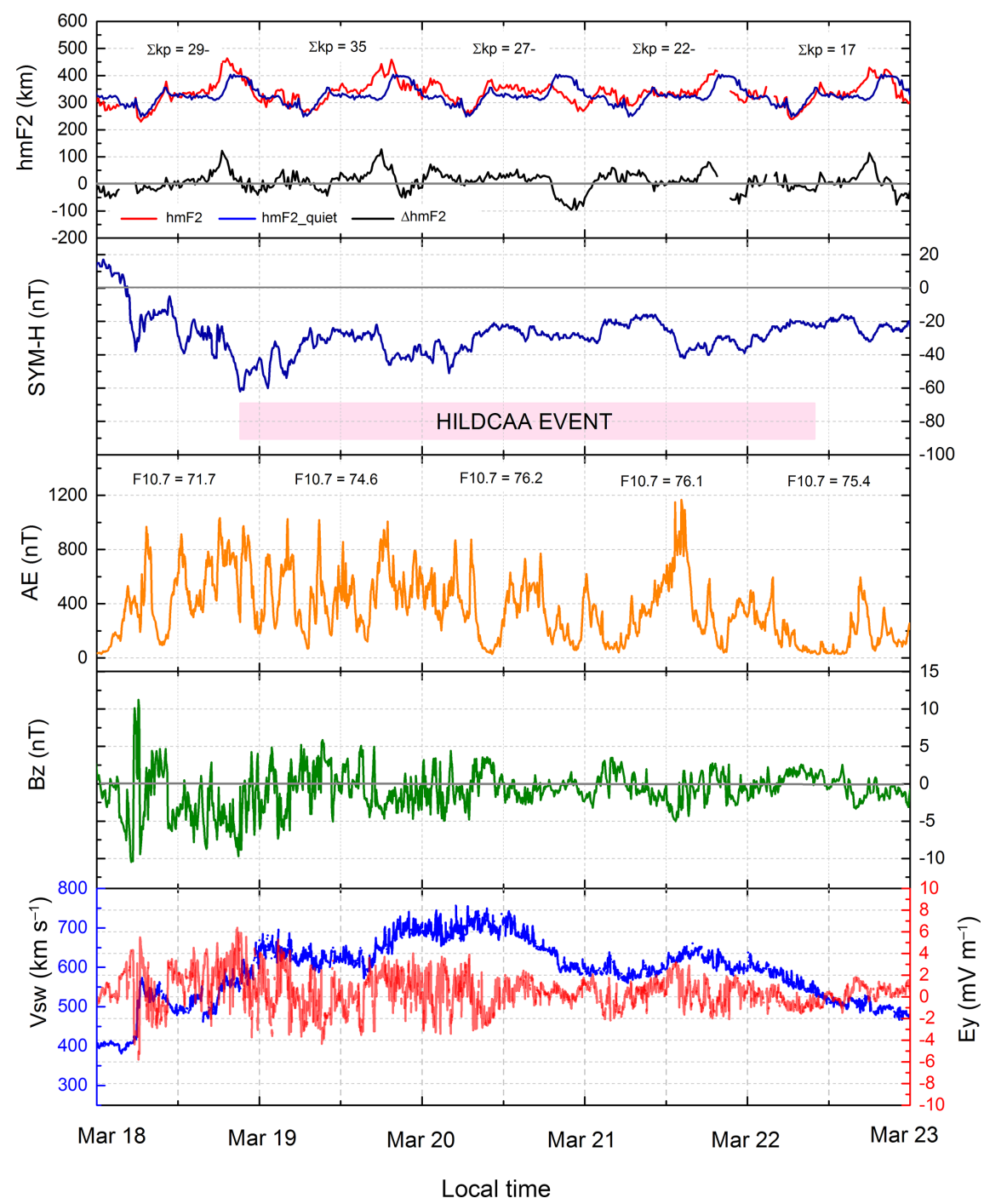

Figure 1. HILDCAA event occurred during 18-22 March 2006 (H-03). From top to bottom: hmF2 (peak height of the F2 layer (red line), the quiet-day period reference (blue line) and $\Delta h m \mathrm{~F} 2$ (black line), SYM-H, AE index, $B_{z}$, and Vsw.

the period and the Vsw reached around $650 \mathrm{~km} \mathrm{~s}^{-1}$. The $E_{y}$ shows high values in the beginning of the event; however, as time goes by the values were decreasing. During this second event the $h m \mathrm{~F} 2$ proved to be far higher than the average of quiet days. The $h m \mathrm{~F} 2$ data gaps were due to either the appearance of a very strong blanketing sporadic E layer or the presence of the critical frequency of the layer below the digisonde low-frequency threshold. An important contribution to the high values of the $\Delta h m \mathrm{~F} 2$ is due to the fact that the PRE presents low values in the solstice of June during the solar minimum. Thus, the difference between disturbed and quiet $h m \mathrm{~F} 2$ values is higher than in the other two events.

Finally, the third event $(\mathrm{H}-12)$ is the most prolonged event of all the events analyzed in the present study, where the event lasted more than 6 days, as shown in Fig. 3. It was realized that this event was preceded by the CIR storm that started on 18 December, as seen in the SYM-H profile. The $\mathrm{AE}$ index peak sometimes exceeded $1000 \mathrm{nT}$, reaching the maximum value on 22 December at 06:10 LT (1538 nT). The peak-to-peak amplitude of $B_{z}$ fluctuations was around $10 \mathrm{nT}$, possibly due to the Alfvén waves. The Vsw remained high throughout the event period (above $700 \mathrm{~km} \mathrm{~s}^{-1}$ ) and the $E_{y}$ presented high oscillations during the first set of days of the event. Regarding the $h m \mathrm{~F} 2$, it does not differ from the calm day conditions. This is one of the HILDCAA features; it presents short or moderate geoeffective disturbances, mainly when the event is long lasting.

\section{Results and discussion}

In this section we will discuss the equatorial ionospheric response to the IEF during the HILDCAA events using a statis- 


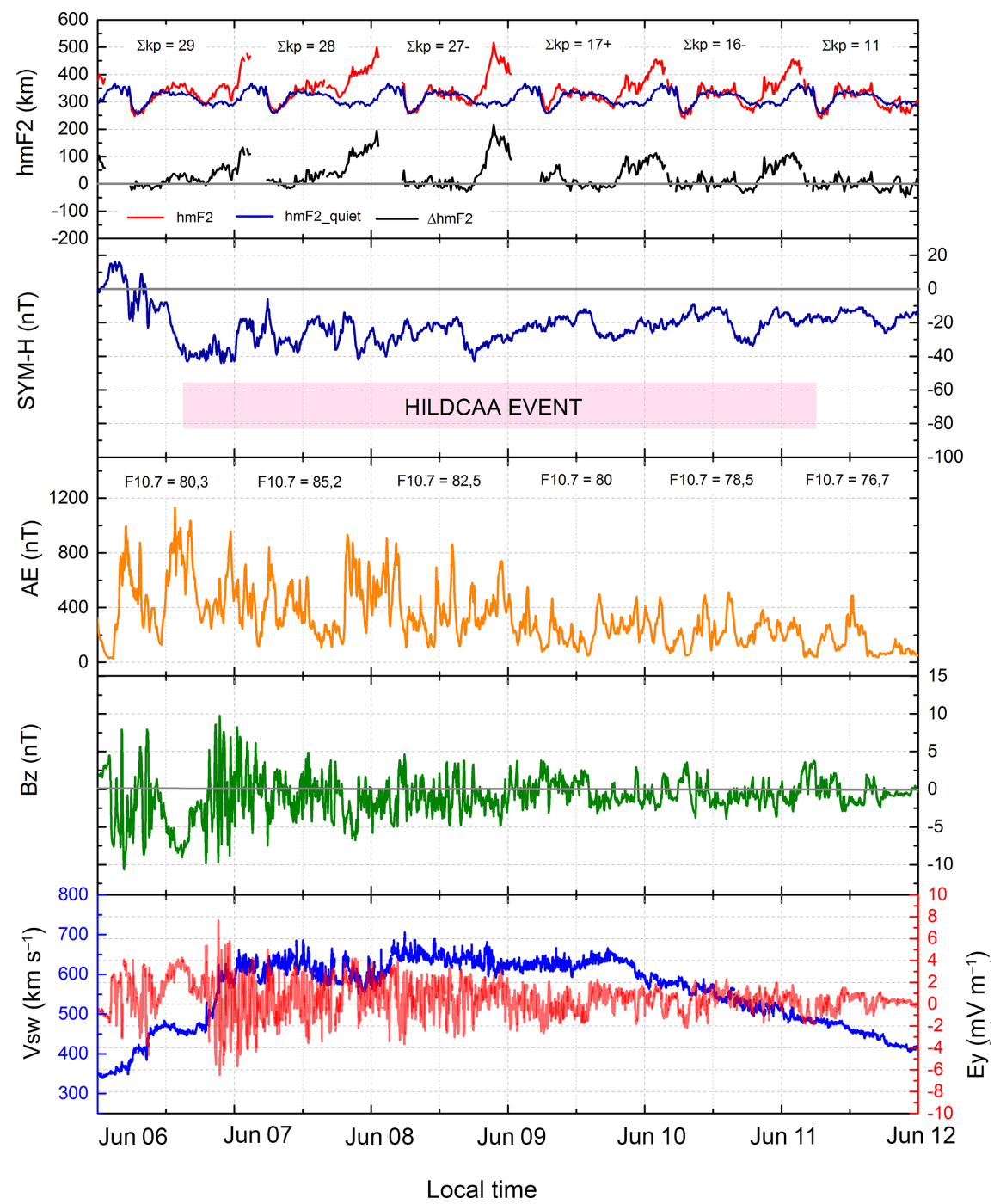

Figure 2. Same as Fig. 1 but for the HILDCAA event that occurred during 6-11 June 2006 (H-06).

tical analysis of the prompt penetration electric fields effects. The correlation analysis between fixed $\Delta h m \mathrm{~F} 2$ values and preceding the $E_{y}$ values for every $15 \mathrm{~min}$ is done using two time intervals, as shown in Fig. 4. This analysis was done for each HILDCAA day as well as for the days before and after the event. Then, the mean value of each time delay was taken. The 4 days before and the 4 days after the event were taken for comparison purposes. The red solid line represents the HILDCAA event during daytime (10 to $12 \mathrm{LT}$ ) and the blue solid line the event during nighttime ( 2 to $4 \mathrm{LT}$ ). The dashed lines are the correlation coefficients for pre-event (rectangle) and post-event (triangle), both during the day (red dotted line) and at night (blue dotted line). The ordinate shows the values of the correlation coefficient, while the abscissa indicates the time delay in decimal hours.

It should be remarked that the correlation coefficient amplitudes was relatively small in all cases here. However, the magnitude of the correlation coefficient is small since the time durations of the southward and northward Alfvénic $B_{z}$ fluctuations are indeed too short to correspond to large correlation coefficients, as it does for the case of geomagnetic storm effects. Therefore, what matters is the overall statistical consistency/regularity of the signal of the correlation coefficient for the respective time period (see Koga et al., 2011).

It was noticed that for H-03 (top panel) the correlation coefficients remained negative during the day and positive during the night, presenting symmetry around correlation zero. Such symmetry is consistent with the fact that the prompt penetrating electric fields present opposite directions considering the local time frame of reference. Comparing the correlation coefficient values for this event, it may be seen that they are smaller for the daytime period than for the nighttime period. This result suggests that the response of the equatorial region with regard to interplanetary electric field penetration is somewhat larger at night. This can be explained by the higher conductivity of the $\mathrm{E}$ region during daytime, which 


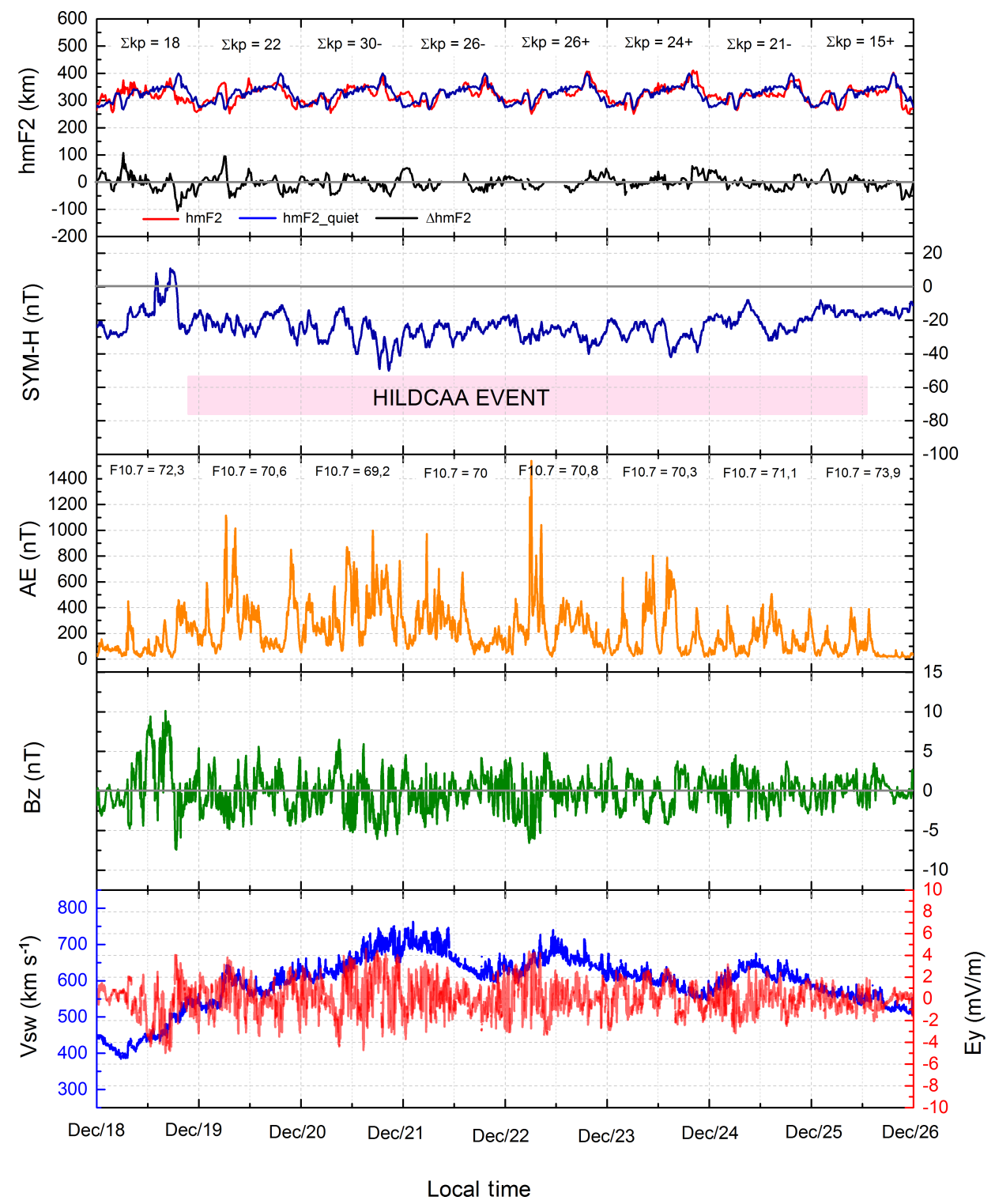

Figure 3. Same as Fig. 1 but for the HILDCAA event that occurred during 18-26 December 2006 (H-12).

partially inhibits the F-region zonal electric fields during the prompt penetration. Since the year 2006 was a descending phase of solar cycle 23, the F10.7 values were small, resulting in an ionosphere less conductive than in the solar maximum periods, but this does not alter the fact that during the day the ionization processes are still effective due to the solar radiation. Consequently, the ionosphere is seen to be more sensitive to the electric field penetration overnight.

When the interplanetary electric field $E_{y}$ is positive (dawn-dusk), the electric field penetration into the equatorial ionosphere is eastward during the day, the correlation between the IEF and equatorial zonal electric field (or F2 peak height) is positive during the day (Wei et al., 2008). However, this is not observed in present study. In the three events studied here, predominantly the correlation is negative (positive) during the day (night). Taking into account the mean values, the peak height of the F2 layer presented a behavior different than expected. Thus, the consequence of these three specifically events in the equatorial ionosphere was the attenuation of the zonal electric field. One interesting case happened during PRE on 20 March, when a clear overshielding event occurred in which $E_{y}$ changed from positive to oscillating around zero (see Fig. 1). This means negative prompt penetration electric field during dayside also causing negative variation in $\Delta h m \mathrm{~F} 2$. As $E_{y}$ oscillates around zero, the correlation between $E_{y}$ and $\Delta h m \mathrm{~F} 2$ is not easy to quantify. This corroborates the explanation for the low correlation values of our results.

An abrupt reversal of $B_{z}$ to southward direction from a steady northward condition causes an undershielding con- 


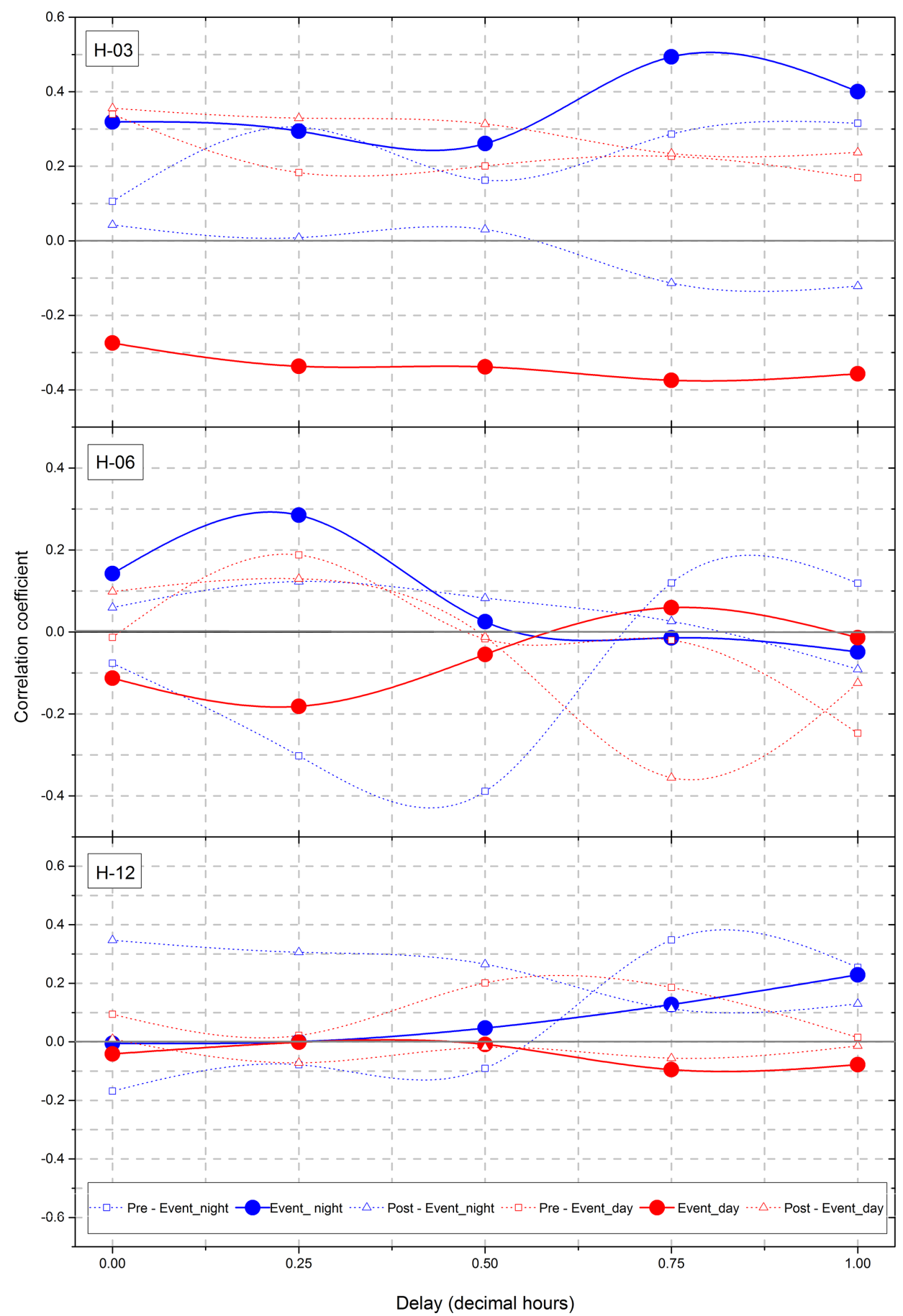

Figure 4. Correlation analysis for three HILDCAA events using two time intervals representative of day (10 to 12 LT) and night (2 to 4 LT) periods. The red lines represent the event during the daytime, while the blue lines represent it during the nighttime. The dashed lines represent pre-event (rectangle) and post-event (triangle), and the solid line represents the event itself.

dition and the region-1 electric field instantaneously penetrates into equatorial and low latitudes. This electric field can operate for between $15 \mathrm{~min}$ and $1 \mathrm{~h}$. Conversely, if, after a steady southward configuration, the $B_{z}$ turns to northward, then overshielding occurs (Forbes, 1995; Kikuchi et al., 1996; Fejer, 1997; Abdu et al., 2006; Fejer et al., 2007; Wolf et al., 2007). If only one abrupt reversal to southward or northward direction occurs, it is classified as a single penetration. When there is an oscillation between northward and southward directions, it is classified as multiple penetrations. 
According to Wei et al. (2008) a multiple electric field penetration is associated with HILDCAA. The electric field coupling process lasts from minutes to hours (Senior and Blanc, 1984; Fejer et al., 1990; Huang et al., 2005), as previously said, but in the present paper delays until 60 min were considered.

The H-06 (central panel) shows correlation change both for the day (from negative to positive) and for the night (from positive to negative) after $30-45 \mathrm{~min}$ of delay. During the day until the first $30 \mathrm{~min}$ of delay the coefficient was negative with low values. During the night the same behavior occurred during the first $30 \mathrm{~min}$ but with positive coefficients. It is well known that, during magnetic storms, the magnetospheric energy input over high latitudes causes heating and upwelling of ionosphere, and, additionally, the disturbance dynamo electric fields dominate the electrodynamical process over middle and low latitudes. This can be seen during HILDCAA events, too. A vertical uplift of the F layer may be seen every day during the event. This result indicates that for the first 30 min of delay the behavior is similar to the $\mathrm{H}-03$ event. For this reason and only for this period, the correlation analysis of the $\mathrm{H}-06$ event presented a predominantly undershielding electric field. The bottom panel shows H-12 with negative correlation coefficients during the day, with very low values, reaching a maximum of -0.1 . During the night the correlation coefficients are positive with very low values or practically zero at the beginning of the analysis; however, they are increasing as the time delay increases. This event was the longest, lasting more than 8 days, and it is worth noting that, due to the duration of the event, its consequences in the ionosphere have reduced the intensity of the penetration effects (Sobral et al., 2006). A weak correlation for this event is due to continuous injection of particles into the ring current. The Dst index is proportional to the kinetic energy of ring current particles, and it may be seen in the second panel of Fig. 3 that during the whole event was low, on average $-22.37 \mathrm{nT}$. As the Dst index is an indicator of geomagnetic activity, this event essentially caused a weak geoeffectiveness in equatorial ionosphere.

All the three events displayed the same behavior: during the daytime the correlation between $E_{y}$ and $\Delta h m \mathrm{~F} 2$ was negative, while it was positive during nighttime. Moreover, in all three cases there is symmetry around correlation equal to zero, which is consistent with the configuration of the prompt penetration electric fields. Note that such symmetry does not occur for the days before and the days after the HILDCAA event. Despite the small values of the correlation coefficients, this methodology was appropriate to observe the behavior of the equatorial and low-latitudinal ionosphere during HILDCAA events. The important point here, as mentioned before, is the statistical regularity of these coefficients. However, a study with a larger number of events is needed to support this statistic.

Given such low correlation coefficients using two time intervals representative of day and night periods, another ap-

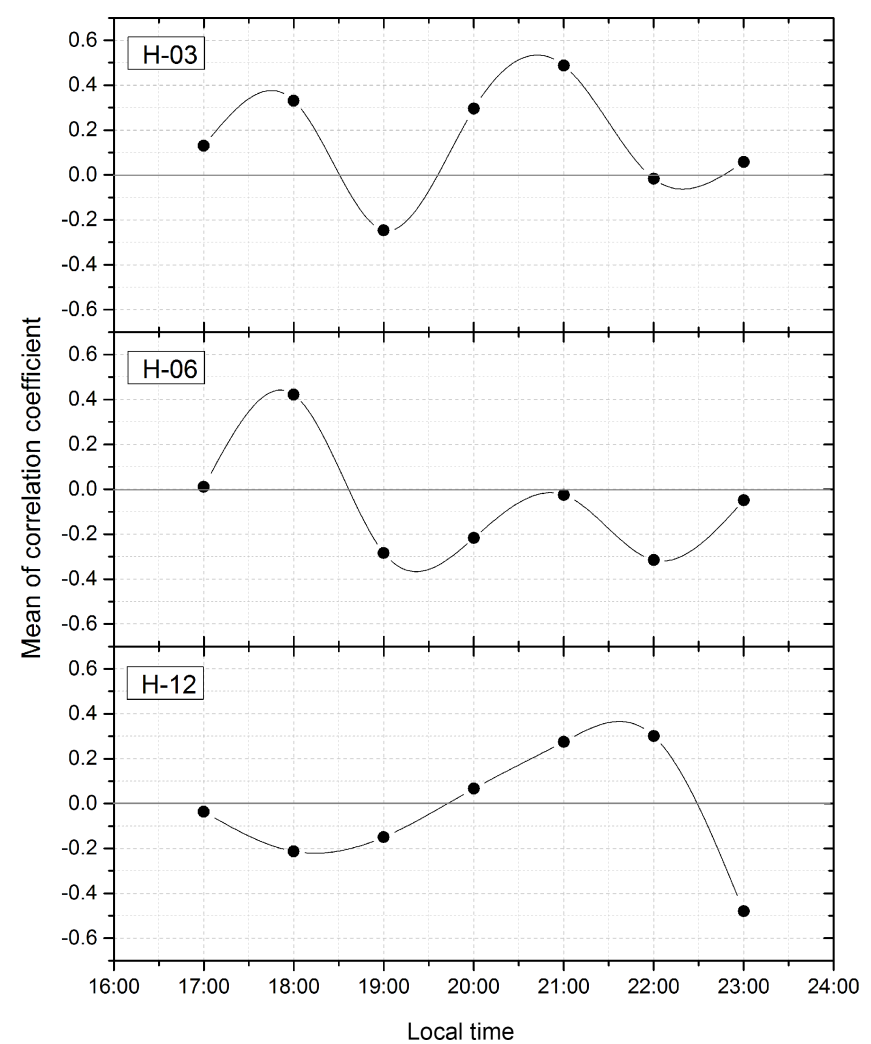

Figure 5. Correlation analysis between $E_{y}$ and $\Delta h m \mathrm{~F} 2$ for the range from 17 to $23 \mathrm{LT}$.

proach was done taking into account the time that precedes and follows the pre-reversal enhancement peak, since the elevation of the layer during the PRE is clearly visible on the days of the events. Figure 5 depicts the correlation analysis between $E_{y}$ and $\Delta h m \mathrm{~F} 2$ around PRE peak, i.e., for the range from 17 to $23 \mathrm{LT}$. Each panel refers to one of the HILDCAA events, distributed as follows: the top panel corresponds to H03 , the central panel to $\mathrm{H}-06$, and the bottom panel to $\mathrm{H}-12$. A cubic spline interpolation was made because the data presented different temporal resolution. After that, the Pearson correlation coefficients were calculated for each individual hour. As each hour provides a value, a representative average was made for that time during the days of the event.

Low values of the correlation coefficients were expected since, during this period, $E_{y}$ has a high oscillation level $( \pm)$ and the ionospheric F-layer height seems to respond to an integrated action $E_{y}$ in time. However, this analysis showed interesting results. The $\mathrm{H}-03$ and $\mathrm{H}-06$ events show very similar behavior to each other, i.e., practically sinusoidal behavior. Both have almost the same duration, with the $\mathrm{AE}$ index remaining quite high throughout the event. The $\mathrm{H}-12$ event is different from the first two, in number of days and intensity of the AE index. This event was not different from the calm day conditions. Another interesting feature that occurred in the $\mathrm{H}-06$ event, and which is different from the others, is due 
to the layer elevation after $24 \mathrm{LT}$, as can be seen in Fig. 2. For this reason greater attention will be devoted to this in future studies. A possible explanation is that $B_{z}$ changes its direction in the transition time in which the electric field changes from positive to negative. That is, an undershielding occurred at the transition time $(\sim 22 \mathrm{LT})$ where the $E_{y}$ changes from positive to negative, followed by an overshielding condition. In fact, just before the transition of the electric field, due to the undershielding raising the layer, it descends with the transition and, when overshielding occurs, the layer returns to rising.

In conclusion, with regard to electric fields in the dusk sector during HILDCAA events, for two first events it was possible to find a kind of relationship between $E_{y}$ and $\Delta h m \mathrm{~F} 2$ under the main focus of the HILDCAA influence. However, it is not possible to associate this with the ionospheric layer elevation only due to HILDCAA effects. Each of the three events displayed a lot of variability, in addition to each event being in a different time of the year and also in different seasons. Furthermore, Sobral et al. (2001) reported that many of disturbed height drifts cannot be explained merely by $B_{z}$ and/or AE time variations and penetration electric fields. Disturbance winds and neutral composition changes can also cause the height of the layer to vary.

\section{Summary and conclusions}

It is well known that the electric field penetration from magnetospheric origin to the equatorial region causes an $h m \mathrm{~F} 2$ variation during the day, while at night the same thing happens but with opposite direction; i.e., the ionosphere rises up during the day at the same time that it falls during the night. This is due to the fact that, no matter the direction of the variation in the electric field, the vertical motion of the F2 layer of the equatorial regions is different for nighttime and daytime periods. However, even though HILDCAAs are perturbed geomagnetic activities, in this work it was noted that the responses of the ionosphere were different than expected.

A major concern about this study was to identify how the equatorial and low-latitude ionosphere over the Brazilian region behaves during HILDCAA events in terms of electric field penetration. On account of this, a study was made in order to see the cross correlation between the penetration of interplanetary electric field and the variation in the F2 layer during HILDCAA events. Initially one representative time range for the daytime and another for nighttime were selected. $\Delta h m \mathrm{~F} 2$ and $E_{y}$ data were high-pass-filtered to allow only effects of direct penetration of electric fields, with a cutoff frequency $9.26 \times 10^{5} \mathrm{~Hz}$. Thereafter, another approach was used to check the correlation of the same data, but only to the times around the PRE peak.

The main results for each of the three events can be summarized as follows:
- Figure 4 shows that the correlation coefficient presents symmetry around correlation coefficient whose pattern is consistent with polarity of the polar cap potential drop (magnetospheric electric field). Moreover, for the preceding and subsequent days such a symmetry ceases to exist, which corroborates the idea of the existence of net prompt penetration electric fields during the HILDCAA events.

- All events studied here showed high variability from overshielding to undershielding electric fields. Nevertheless, one statistical study with a larger number of events will be necessary to be able to confirm the prevalence of each of them during HILDCAA occurrence.

- Figure 5 presents the HILDCAA influence from 17 to 23 LT, i.e., involving the PRE peak. Despite mean values not being very high, it is important note the quite similar behavior of the events of March and June. This behavior may be perhaps a signal pattern; however, a larger study covering more HILDCAA events is needed to support this hypothesis.

To conclude, the assessment accepted by the authors is that there is clear evidence of prompt penetration electric fields during the HILDCAA events, with their geoeffectiveness (height variations in the F2 layer) being of relatively small amplitude, with the exception of times around the PRE peak, when the HILDCAA is seen to contribute to the elevation of the ionosphere.

Data availability. The OMNI data were obtained from the GSFC/SPDF OMNIWeb interface at https://omniweb.gsfc.nasa. gov. The Kp data were obtained from the World Data Center for Geomagnetism, Kyoto, at http://wdc.kugi.kyoto-u.ac.jp/. The F10.7 data were obtained from National Geophysical Data Center (NGDC) at http://spidr.ionosonde.net/spidr/. The digisonde data used in this study may be acquired by contacting the coordinator responsible at DAE/INPE (Inez S. Batista, e-mail: inez.batista@inpe.br).

Competing interests. The authors declare that they have no conflict of interest.

Special issue statement. This article is part of the special issue "Space weather connections to near-Earth space and the atmosphere". It is a result of the $6^{\circ}$ Simpósio Brasileiro de Geofísica Espacial e Aeronomia (SBGEA), Jataí, Brazil, 26-30 September 2016. 
Acknowledgements. Regia Pereira Silva acknowledges the support from Conselho Nacional de Desenvolvimento Científico e Tecnológico (CNPq) through grant no. 140788/2015-8. Jose Humberto Andrade Sobral, Daiki Koga, and Jonas Rodrigues de Souza would like to acknowledge the financial support from CNPq process numbers 303741, 112886/2015-9, and 305885/2015-4, respectively. The authors thank DAE/INPE for kindly providing the digisonde data.

The topical editor, Ricardo A. Buriti, thanks Rajkumar Hajra and one anonymous referee for help in evaluating this paper.

\section{References}

Abdu, M. A., Bittencourt, J. A., and Batista, I. S.: Magnetic declination control of the equatorial $\mathrm{F}$ region dynamo electric field development and spread F, J. Geophys. Res., 86, 11443-11446, 1981.

Abdu, M. A., Batista, I. S., Walker, G. O., Sobral, J. H. A., Trivedi, N. B., and De Paula, E. R.: Equatorial ionospheric electric fields during magnetospheric disturbances: local time/longitude dependences from recent EITS campaigns, J. Atmos. Sol.-Terr. Phy., 57, 1065-1083, 1995.

Abdu, M. A., Sastri, J. H., MacDougall, J., Batista, I. S., and Sobral, J. H. A.: Equatorial disturbance dynamo electric field longitudinal structure and spread F: a case study from GUARA/EITS campaigns, J. Geophys. Res., 24, 1707-1710, 1997.

Abdu, M. A., MacDougall, J. W., Batista, I. S., Sobral, J. H. A., and Jayachandran, P. T.: Equatorial evening prereversal electric field enhancement and sporadic $\mathrm{E}$ layer disruption: A manifestation of E and F region coupling, J. Geophys. Res., 108, 1254, https://doi.org/10.1029/2002JA009285, 2003.

Abdu, M. A., De Souza, J. R., Sobral, J. H. A., and Batista, I. S.: Magnetic storm associated disturbance dynamo effects in the low and equatorial latitude ionosphere, in: Recurrent magnetic storms: corotating solar wind Streams, edited by: Tsurutani, B., McPherron, R., Gonzalez, W., Lu, G., Sobral, J. H. A., and Gopalswamy, N., Washington, DC: American Geophysical Union, 283-304, AGU Code GM1674327, Geophysical Monograph Series, Vol. 167, 2006.

Alves, M. V., Echer, E., and Gonzalez, W. D.: Geoeffectiveness of corotating interaction regions as measured by Dst index, J. Geophys. Res., 111, A07S05, https://doi.org/10.1029/2005JA011379, 2006.

Baker, D. N., Blake, J. B., Klebesadel, R. W., and Higbie, P. R.: Highly relativistic electrons in the Earth's outer magnetosphere: 1. Lifetimes and temporal history 1979-1984, J. Geophys. Res., 91, 4265-4276, https://doi.org/10.1029/JA091iA04p04265, 1986.

Basu, B.: Generalized Rayleigh-Taylor instability in the presence of time-dependent equilibrium, J. Geophys. Res., 102, 17305$17312,1997$.

Blanc, M. and Richmond, A. D.: The ionospheric disturbance dynamo, J. Geophys. Res., 85, 1669-1686, 1980.

Borovisky, J. E. and Denton, M. H.: Differences between CMIdriven storms and CIR-driven storms, J. Geophys. Res., 111, A07S08, https://doi.org/10.1029/2005JA011447, 2006.
Fejer, B. G.: The electrodynamics of the low-latitude ionosphere: recent results and future challenges, J. Atmos. Sol.-Terr. Phy., 59, 1465-1482, 1997.

Fejer, B. G. and Scherliess, L.: Empirical models of storm time equatorial zonal electric fields, J. Geophys. Res., 102, 2404724056, 1997.

Fejer, B. G., Spiro, R. W., Wolf, R. A., and Foster, J. C.: Latitudinal variation of perturbation electric fields during magnetically disturbed periods: 1986 SUNDIAL observations and model results, Ann. Geophys., 8, 441-454, 1990.

Fejer, B. G., Jensen, J. W., Kikuchi, T., Abdu, M. A., and Chau, J. L.: Equatorial Ionospheric Electric Fields During the November 2004 Magnetic Storm, J. Geophys. Res., 112, A10304, https://doi.org/10.1029/2007JA012376, 2007.

Forbes, J. M.: Equatorial penetration of magnetic disturbance effects in the thermosphere and ionosphere, J. Atmos. Sol.-Terr. Phy., 57, 1085-1093, 1995.

Gonzalez, W., Tsurutani, B. T., and Clua De Gonzalez, A. L.: Interplanetary origin of geomagnetic storms, Space Sci. Rev., 88, 529-562, 1999.

Gonzalez, W. D., Guarnieri, F. L., Clua-Gonzalez, A. L., Echer, E., Alves, M. V., Ogino, T., and Tsurutani, B. T.: Magnetospheric energetics during HILDCAAs, Recurrent Magnetic Storms: Corotating Solar Wind Streams, 175-182, 2006.

Gosling, J. T. and Pizzo, V. J.: Formation and evolution of corotating interaction regions and their three dimensional structure, in: Corotating Interaction Regions, 21-52, Springer Netherlands, 1999.

Guarnieri, F. L.: The nature of auroras during high-intensity longduration continuous AE activity (HILDCAA) events: 19982001, in: Recurrent Magnetic Storm: Corotating Solar Wind Strems, edited by: Tsurutani, B., McPherron, R., Gonzalez, W., Lu, G., Sobral, J. H. A., and Gopalswamy, N., Geophys. Monogr. Ser., Vol. 167, 235 pp., AGU, Washington, D. C., https://doi.org/10.1029/167GM19, 2006.

Hajra, R., Echer, E., Tsurutani, B. T., and Gonzalez, W. D.: Solar cycle dependence of High-Intensity Long-Duration Continuous AE Activity (HILDCAA) events, relativistic electron predictors?, J. Geophys. Res., 118, 5626-5638, https://doi.org/10.1002/jgra.50530, 2013.

Hajra, R., Echer, E., Tsurutani, B. T., and Gonzalez, W. D.: Superposed epoch analyses of HILDCAAs and their interplanetary drivers: Solar cycle and seasonal dependences, J. Atmos. Sol.Terr. Phy., 121, 24-31, 2014a.

Hajra, R., Echer, E., Tsurutani, B. T., and Gonzalez, W. D.: Relativistic electron acceleration during high-intensity longduration, continuous AE activity (HILDCAA) events: Solar cycle phase dependences, Geophys. Res. Lett., 41, 1876-1881, https://doi.org/10.1002/2014GL059383, 2014b.

Hajra, R., Echer, E., Tsurutani, B. T., and Gonzalez, W. D.: Solar wind-magnetosphere energy coupling efficiency and partitioning: HILDCAAs and preceding CIR storms during solar cycle 23, Geophys. Res. Lett., 119, 2675-2690, https://doi.org/10.1002/2013JA019646, 2014c.

Hajra, R., Tsurutani, B. T., Echer, E., Gonzalez, W. D., Brum, C. G. M., Vieira, L. E. A., and Santolik, O.: Relativistic electron acceleration during HILDCAA events: are precursor CIR magnetic storms important?, Earth Planets Space, 67, 109, https://doi.org/10.1186/s40623-015-0280-5, 2015. 
Huang, C. M., Richmond, A. D., and Chen, M.-Q.: Theoretical effects of geomagnetic activity on low-latitude ionospheric electric fields, J. Geophys. Res., 110, A05312, https://doi.org/10.1029/2004JA010994, 2005.

Kahler, S.: Coronal mass ejections, Rev. Geophys., 25, 663-675, 1987.

Kelley, M. C.: The Earth's Ionosphere, New York, Academic Press, 1989.

Kelley, M. C., Ilma, R. R., and Crowley, G.: On the origin of pre-reversal enhancement of the zonal equatorial electric field, Ann. Geophys., 27, 2053-2056, https://doi.org/10.5194/angeo27-2053-2009, 2009.

Kikuchi, T., Lühr, H., Kitamura, T., Saka, O., and Schlegel, K.: Direct penetration of the polar electric field to the equator during a DP 2 event as detected by the auroral and equatorial magnetometer chains and the EISCAT radar, J. Geophys. Res., 101, 1716117173, https://doi.org/10.1029/96JA01299, 1996.

Kim, H.-J.: Study on the particle injections during HILDCAA intervals, J. Astron. Space Sci., 24, 119-124, 2007.

Koga, D., Sobral, J. H. A., Gonzalez, W. D., Arruda, S. C. S., Abdu, M. A., Castilho, V. M., Mascarenhas, M., Gonzalez, A. C., Tsurutani, B. T., Denardini, C. M., and Zamlutti, C. J.: Electrodynamic coupling process between the magnetosphere and the equatorial ionosphere during a 5-day HILDCAA event, J. Atmos. Sol.-Terr. Phy., 73, 148-155, 2011.

Kozyra, J. U., Crowley, G., Emery, B. A., Fang, X., Maris, G., Mlynczak, M. G., and Rong, P. P.: Response of the upper/middle atmosphere to coronal holes and powerful high-speed solar wind streams in 2003, Recurrent Magnetic Storms: Corotating Solar Wind Streams, 319-340, 2006.

Meredith, N. P., Cain, M., Horne, R. B., Thorne, R. M., Summers, D., and Anderson, R. R.: Evidence for chorus-driven electron acceleration to relativistic energies from a survey of geomagnetically disturbed periods, J. Geophys. Res., 108, 1248, https://doi.org/10.1029/2002JA009764, 2003.

Paulikas, G. A. and Blake, J. B.: Effects of the Solar Wind on Magnetospheric Dynamics: Energetic Electrons at the Synchronous Orbit, in: Quantitative Modeling of Magnetospheric Processes, edited by: Olson, W. P., American Geophysical Union, Washington, D. C., https://doi.org/10.1029/GM021p0180, 1979.

Richardson, I. G.: Energetic particles and corotating interaction regions in the solar wind, Space Sci. Rev., 111, 267-376, 2004.

Richmond, A. D., Peymirat, C., and Roble, R. G.: Longlasting disturbances in the equatorial ionospheric electric field simulated with a coupled magnetosphere-ionospherethermosphere model, J. Geophys. Res., 108, 1118, https://doi.org/10.1029/2002JA009758, 2003.

Sandanger, M. I., Soraas F., Aarsnes, K., Oksavik, K., Evans, D. S., and Greer, M. S.: Proton injections into the ring current associates with $B_{z}$ variations during HILDCAA events, in: The Inner Magnetosphere: Physics and Modeling, edited by: Pulkkinen, T. I., Tsyganenko, N. A., and Friedel, R. H. W., Geophys. Monogr. Ser., Vol. 155, p. 249, AGU, Washington, D.C., 2005.

Santos, A. M., Abdu, M. A., Souza, J. R., Sobral, J. H. A., and Batista, I. S.: Disturbance zonal and vertical plasma drifts in the Peruvian sector during solar minimum phases, J. Geophys. Res., 121, 2503-2521, 2016.

Sastri, J. H.: Equatorial electric-fields of ionospheric disturbance dynamo origin, Ann. Geophys., 6, 635-642, 1988.
Senior, C. and Blanc, M.: On the control of magnetospheric convection by the spatial distribution of ionospheric conductivities, J. Geophys. Res., 89, 261-284, https://doi.org/10.1029/JA089iA01p00261, 1984.

Smith, E. J. and Wolfe, J. H.: Observations of interaction regions and corotating shocks between one and five AU: Pioneers 10 and 11, Geophys. Res. Lett., 3, 137-140, 1976.

Sobral, J. H. A., Abdu, M. A., Gonzalez, W. D., Batista, I., and de Gonzalez, A. C.: Low-latitude ionospheric response during intense magnetic storms at solar maximum, J. Geophys. Res. 102, 14305-14313, 1997.

Sobral, J. H. A., Abdu, M. A., Yamashita, C. S., Gonzalez, W. D., Clua De Gonzalez, A. L., Batista, I. S., Zamlutti, C. J., and Tsurutani, B. T.: Responses of the low-latitude ionosphere to very intense geomagnetic storms, J. Atmos. Sol.-Terr. Phy., 63, 965974, 2001.

Sobral, J. H. A., Abdu, M. A., Gonzalez, W. D., Clua De Gonzalez, A. L., Tsurutani, B. T., Da Silva, R. R. L, Barbosa, I. G., Arruda, D. C. S., Denardini, C. M., Zamlutti, C. J., and Guarnieri, F.: Equatorial ionospheric responses to high-intensity long-duration auroral electrojet activity (HILDCAA), J. Geophys. Res., 111, A07S02, https://doi.org/10.1029/2005JA011393, 2006.

Soraas, F., Aarsn, K., Oksavik, K., Sandanger, M. I., Evans, D. S., and Greer, M. S.: Evidence for particle injection as the cause of Dst reduction during HILDCAA events, J. Atmos. Sol.-Terr. Phy., 66, 177-186, 2004.

Summers, D., Thorne, R. M., and Xiao, F.: Relativistic theory of wave-particle resonant diffusion with application to electron acceleration in the magnetosphere, J. Geophys. Res., 103, 2048720500, https://doi.org/10.1029/98JA01740, 1998.

Tsurutani, B. T. and Gonzalez, W. D.: The cause of high intensity long-duration continuous AE activity (HILDCAA): interplanetary Alfvén wave trains, Planet Space Sci., 35, 405-412, 1987.

Tsurutani, B. T. and Gonzalez, W. D.: The interplanetary causes of magnetic storms: a review. Magnetic storms, Geophysical Monograph Series, 98, 77-89, 1997.

Tsurutani, B. T., Ho, C. M., Arballo, J. K., Goldstein, B. E., and Balogh, A.: Large amplitude IMF fluctuations in Corotating interaction regions: Ulysses at midlatitudes, J. Geophys. Res., 22, 3397-3400, 1995.

Tsurutani, B. T., Gonzalez, W. D., Guarnieri, F., Kamide, Y., Zhou, X., and Arballo, J. K.: Are high-intensity long-duration continuous AE activity (HILDCAA) events substorm expansion events?, J. Atmos. Sol.-Terr. Phy., 66, 167-176, 2004.

Tsurutani, B. T., Gonzalez, W. D., Gonzalez, A. L. C., Guarnieri, F. L., Gopalswamy, N., Grande, M., Kamide, Y., Kasahara, Y., Lu, G., Mann, I., McPherron, R., Soraas, F., and Vasyliunas, V.: Corotating solar wind streams and recurrent geomagnetic activity: A review, J. Geophys. Res., 111, A07S01, https://doi.org/10.1029/2005JA011273, 2006a.

Tsurutani, B. T., McPherron, R. L., Gonzalez, W. D., Lu, G., Gopalswamy, N., and Guarnieri, F. L.: Magnetic storms caused by corotating solar wind streams, in: Recurrent Magnetic Storms: Corotating Solar Wind Streams, edited by: Tsurutani, B., McPherron, R. L., Gonzalez, W. D., Lu, G., Sobral, J. H. A., and Gopalswamy, N., Geophysical Monograph Series, Vol. 167, AGU, Washington, D.C., 1-17, 2006 b.

Tsurutani, B. T., Horne, R. B., Pickett, J. S., Santolik, O., Schriver, D., and Verkhoglyadova, O. P.: Introduction 
to the special section on Chorus: Chorus and its role in space weather, J. Geophys. Res.-Space, 115, A00F01, https://doi.org/10.1029/2010JA015870, 2010.

Tsurutani, B. T., Echer, E., Guarnieri, F. L., and Gonzalez, W. D.: The properties of two solar wind high speed streams and related geomagnetic activity during the declining phase of solar cycle 23, J. Atmos. Sol.-Terr. Phy., 73, 167-177, 2011a.

Tsurutani, B. T., Laknina, G. S., Verkhoglyadova, O. P., Gonzalez, W. D., Echer, E., and Guarnieri, F. L.: A review of interplanetary discontinuities and their geomagnetic effects, J. Atmos. Sol. Terr. Phy., 73, 5-19, 2011 b.

Turner, N. E., Mitchell, E. J., Knipp, D. J., and Emery, B. A.: Energetics of magnetic storms driven by corotating interaction regions: a study of geoeffectiveness, in: Recurrent Magnetic Storms, edited by: Tsurutani, B., McPherron, R., Gonzalez, W., Lu, G., Sobral, J. H. A., and Gopalswamy, N., Corotating Solar Wind Streams, 167, American Geophysical Union Press, Washington, DC, 113 pp., 2006.
Wagner, W. J.: Coronal mass ejections, Annu. Rev. Astron. Astr., 22, 267-289, 1984.

Watari, S.: The effect of the high-speed stream following the corotating interaction region on the geomagnetic activities, Ann. Geophys., 15, 662-670, https://doi.org/10.1007/s00585-997-0662-5, 1997.

Webb, D. F. and Howard, T. A.: Coronal mass ejections: Observations, Living Reviews in Solar Physics, 9, 1-83, 2012.

Wei, Y., Hong, M., Wan, W., Du, A., Lei, J., Zhao, B. Wang, W., Ren, Z., and Yue, X.: Unusually long lasting multiple penetration of interplanetary electric field to equatorial ionospheric under oscillating IMF $B_{z}$, J. Geophys. Res., 35, L02102, https://doi.org/10.1029/2007GL032305, 2008.

Wolf, R. A., Spiro, R. W., Sazykin, S., and Toffoletto, F. R.: How the Earth's inner magnetosphere works: An evolving picture, J. Atmos. Sol.-Terr. Phy., 69, 288-302, 2007. 